\title{
PROSPECTS FOR THE APPLICATION OF A MOBILE VLBI SYSTEM IN CHINA
}

\author{
Cai Jianqing and Xia Hanlin \\ $\mathrm{Xi}^{\prime}$ an Institute of Surveying and Mapping \\ No.1 Mid-Yanta Road, Xi'an 710054, P.R.China
}

\begin{abstract}
Based on the present status and projects of Chinese VLBI and SLR fixed stations, the applications of a mobile VLBI system are investigated in establishing a Chinese high accuracy geodetic VLBI network and terrestrial reference frame.
\end{abstract}

\section{INTRODUCTION}

Since Very Long Baseline Interferometry (VLBI) was first used in geodetic application in 1972 , considerable progress has been made over the past two decades. The precision of VLBI baseline measurements has been improved by two orders of magnitude from about 1 meter to $<1 \mathrm{~cm}$. VLBI has now become one of the most powerful space geodetic tools in establishing a high accuracy geodetic control network and global terrestrial reference frame, determining earth orientation parameters and monitoring plate motion and regional crustal deformation.

In China, the Chinese Academy of Science began to undertake a Chinese VLBI network project in 1979, which would consist of three fixed VLBI stations and a data analysis center. The Shanghai $25-\mathrm{m}$ antenna VLBI station has been operated since 1987. The Urumqi station with a 25-m antenna is under construction and will be operational in 1993. The Kunming station is planned. The data analysis center, operated by the Shanghai Observatory, is nearly completed (Ye Shuhua et al., 1992). In addition, a mobile VLBI station will be constructed.

In this paper, we study the applications of a mobile VLBI system in establishing a Chinese geodetic VLBI network and terrestrial reference frame. Recommendations for further developments and applications of VLBI and SLR techniques in China are also given.

\section{PROSPECTS FOR THE APPLICATION OF A MOBILE VLBI IN CHINA}

The proposed Chinese mobile VLBI system (CMV-1) would consist of a 4 $5 \mathrm{~m}$ collapsible antenna, a dual-frequency $\mathrm{S} / \mathrm{X}$ band receiver, a data acquisition system compatible with MK III (A) system and a hydrogen maser standard. Considering the possible upgrades of VLBI technique in the 1990's, such as wider bandwidths and higher recording speeds (Ray, 1991), CMV-1 is designed for the possibility of improvement and extension.

Mobile VLBI has many applications which have been discussed in detail by Clark et al.

421

I.I. Mueller and B. Kotaczek (eds.),

Developments in Astrometry and Their Impact on Astrophysics and Geodynamics, 421-426.

(C) 1993 IAU. Printed in the Netherlands. 
[1985], Davidson et al. [1985], Clark et al. [1987] and Ma et al. [1990]. Considering the particular conditions of China, $\mathrm{CMV}-1$ is deemed appropriate for three major applications:

* To participate in establishing the Chinese National Space Geodetic Network.

* To establish and maintain a regional terrestrial reference frame.

* To determine tectonic plate motion and monitor crustal deformation in China.

\subsection{The Establishment of the Chinese National Space Geodetic Network}

As stated in the introduction, the number of fixed VLBI stations in China is too small to meet the requirements for establishing the Chinese National Space Geodetic Network. It is necessary to use a mobile VLBI system together with three fixed VLBI stations to lay out a fiducial network--geodetic VLBI network, which will provide the primary framework for the national network.

The geodetic VLBI network will consist of 20 to 30 stations, which are distributed throughout China. The locations of network sites are shown in Figure 1, selected according to the following criteria:

* Include all fixed VLBI and SLR stations.

* Include four GPS satellite tracking stations.

* A number of sites will be located in the border regions of China, so as to strengthen geodetic control of the national network in these areas.

* Considering the uniformity of site distribution and traffic conditions to these sites.

In the observation program of this network, all three fixed VLBI stations would participate in observations with each mobile VLBI site. Observing sessions of 48 hours would be used and 10 to 15 sources with adequate strength would be observed. A variance-covariance analysis program was used to optimize an observation schedule which is applicable to this network and to estimate the accuracies of relevant parameters. The results show that baseline accuracies would range from $10^{-8} \sim 10^{-9}$ and site position accuracies would be better than $3 \mathrm{~cm}$.

The geodetic VLBI network as the primary framework together with the GPS densification network would establish the National Space Geodetic Network with relative position accuracy of $10^{-6} \sim 10^{-8}$.

\subsection{The Establishment and Maintenance of a Regional Terrestrial Reference Frame}

Several activities for establishing and maintaining the terrestrial reference frame are now being undertaken by a number of agencies. A terrestrial reference frame with centimeter level accuracy could be defined by the use of measurements available from VLBI and SLR. With improvements in VLBI and SLR techniques over the next few years, the accuracy of the frame would approach the millimeter level. 
In China, the Shanghai third generation VLBI and SLR stations and the Wuhan SLR station have participated in these activities and achieved high accuracy station positions in the global frame. Several new fixed and mobile VLBI and SLR stations (shown in Figure 1) will be constructed in the near future. All of these lay the foundation of the regional terrestrial reference frame.

The establishment and maintenance of the regional frame would be very useful in the independent determination of GPS satellite orbits. This is of great importance to the densifying of the National Space Geodetic Network and the study of geodynamics in China. On the other hand, owing to the fact that sites of the global terrestrial reference frame ( such as ITRF91 recently published by IERS (1992)) are distributed unhomogeneously and concentrated in North America and Europe, these frames are not perfect to some extent. Establishment and maintenance of the regional frame would also contribute to the improvement of the global coverage and stability of the global terrestrial reference frames.

From Figure 1, we see that most fixed VLBI and SLR stations are located in eastern China. To fulfill the establishment of the regional frame, mobile VLBI and SLR stations are necessary. In the following sections, the role of mobile VLBI in these activities will be discussed in detail.

\subsubsection{The Optimal Regional Terrestrial Reference Frame by VLBI}

The considerations for establishing an optimal frame by VLBI include the number and distribution of sites. First, a considered original set of 20 sites was chosen from the 28 sites of the geodetic VLBI network according to the condition of having uniform distribution in mainland China. Of these 20 sites, five fixed VLBI and SLR stations ( Shanghai, Urumqi, Kunming, Changchun and $\mathrm{Xi}^{\prime}$ an ) would always be included among the optimal stations.

We begin with the observational equations used to establish a terrestrial reference frame (Mueller, 1989), the set of stations defining the optimal frame could be chosen according to the criterion of the minimizing the trace of the normal equation matrix inverse computed from the observational equations (Kosek et al., 1990).

Based on an analysis of the selected set of sites using the method mentioned above, we have concluded that $10 \sim 15$ well-distributed stations having high accuracy 3-D coordinates can establish an optimal terrestrial reference frame in China with an accuracy of several centimetres. The maintenance of the frame would be performed by repeated occupation of these sites using the mobile VLBI station.

\subsubsection{The Establishment of a Regional VLBI / SLR Reference Frame and Its Application}

Using collocation observations made with the mobile VLBI system alongside the four fixed 
SLR stations at Changchun, Xi'an, Wuhan and Beijing, together with the existing three fixed VLBI / SLR collocation sites, the transformation parameters between the VLBI and SLR reference frames can be obtained. As a result, a combined VLBI/SLR terrestrial reference frame that takes the advantage of both techniques can be established in China.

It is obvious that the VLBI / SLR reference frame has important implications for some applications, such as precise tracking of GPS satellites.

Since 1990, the experiment of determining precise GPS satellite orbits has been performed every year by use of tracking data from four GPS stations, located in Shanghai, Urumqi, Kunming and Changchun. The primary solution demonstrates that the orbit determination accuracy corresponds to the GPS broadcast ephemerides $(20 \sim 100 \mathrm{~m})$. The main error sources are the GPS antenna position error and pseduo-range measurement error. The above four tracking sites are located near VLBI / SLR sites; so the coordinates of the GPS receiver antennas can be established in the regional VLBI/SLR frame by local survey with an accuracy of better than $10 \mathrm{~cm}$. The GPS satellite orbit can be estimated with an accuracy of $1 \sim 2 \mathrm{~m}$. The effect of orbit error $|\mathrm{dr}|$ on geodetic baselines can be approximated by

$$
\frac{|d L|}{L} \approx \frac{|d r|}{r},
$$

where $|\mathrm{dL}|$ is the error on a baseline of length $\mathrm{L}$ and $\mathrm{r}$ is the range to the satellite. Equation 1 suggests that an error of $1 \sim 2 \mathrm{~m}$ in the orbit will cause a $10^{-7}$ relative baseline error, which would meet the accuracy requirement for establishing the National Space Geodetic Network. In addition, the collocation of four GPS tracking stations with the VLBI / SLR sites will facilitate the independent determination of transformation parameters between the WGS84 and VLBI / SLR reference frames in China.

\section{CONCLUSIONS AND RECOMMENDATIONS}

Based on the discussion of this paper, we have the following conclusions and recommendations:

* A geodetic VLBI network with a baseline accuracy of $10^{-8} \sim 10^{-9}$ and a site position accuracy of better than $3 \mathrm{~cm}$ can be established by use of mobile and fixed VLBI stations, which will provide the primary framework for the National Space Geodetic Network.

* $10 \sim 15$ well-distributed stations having high accuracy 3-D coordinates can establish an optimal regional terrestrial reference frame in China with an accuracy of several centimetres.

* A regional VLBI/SLR reference frame can be established by the collocation observations of mobile and fixed VLBI stations alongside seven fixed SLR sites. The accuracy of GPS satellite orbit determination can be improved from $20 \sim 100 \mathrm{~m}$ to 
$1 \sim 2 \mathrm{~m}$ with the data available from four GPS tracking stations whose coordinates have been obtained in the regional VLBI / SLR frame.

* The development of two or more mobile VLBI stations is helpful in strengthening the observation ability of mobile VLBI to establish and maintain the geodetic VLBI network and regional terrestrial reference frame. They can also be used to check the reliability of mobile VLBI measurements.

* Chinese VLBI and SLR stations should participate in international activities as much as possible in order to establish the accurate connection of the regional VLBI / SLR reference frame with the global frame. This would also contribute to the improvement of the global coverage and stability of the global terrestrial reference frames.

ACKNOWLEDGMENTS The authors would like to acknowledge Professor Wei Ziqing and Dr. Ma Chopo for reading over the manuiscript with critical and instructive suggest and to Dr. Lowe of JPL for going over the manuiscript.

\section{REFERENCES}

[1] Clark, T. A. et al., Precision Geodesy Using the Mark-III Very Long Baseline Interferometer System, IEEE Trans. Geosci. Remote Sensing,Vol. GE-23, No. 4, 438-449, July 1985.

[2] Clark, T. A. et al., Determination of Relative Site Motion in the Western United States Using Mark III Very Long Baseline Interferometer, JGR, Vol. 92, No. B12, 12741-12750,1987.

[3] Davidson, J. and Trask, D.W., Utilization of Mobile VLBI for Geodetic Measurements, ibid [1], 426-437, 1985.

[4] IERS, 1991 IERS Annual Report, Observatoire de Paris, 1992.

[5] Kosek, W. and Kolaczek, B., the Optimum Coventional Terrestrial System Determined by VLBI and SLR Stations, in Earth Rotation and Coordinate Reference Frames, International Association of Geodesy Symposia 105, August 10-11, 1989,138-145, Springer-Verlag, New York / Berlin.

[6] Ma. C. et al., Measurement of Horizontal Motions in Alaska Using Very Long Baseline Interferometry, J.Gcophys. Res., VoL.95, No.B12, 21991-22011, 1990.

[7] Mueller, I.I., Reference Coordinate Systems: A Update, in Theory of Satellite Geodesy and Gravity Field Determination, Sanso, F. and Rummel, R.(Eds.), 153-196, Springer-Verlag, 1989.

[8] Ray, J.R., Radio Interferometry, Review of Gcophysics Supplement 148-156, 1991.

[9] Ye Shuhua et al., Chinese Crustal Motion and Geodynamics Project, SGMS Newsletter, Vol.3, No.1, 23-27, 1992. 


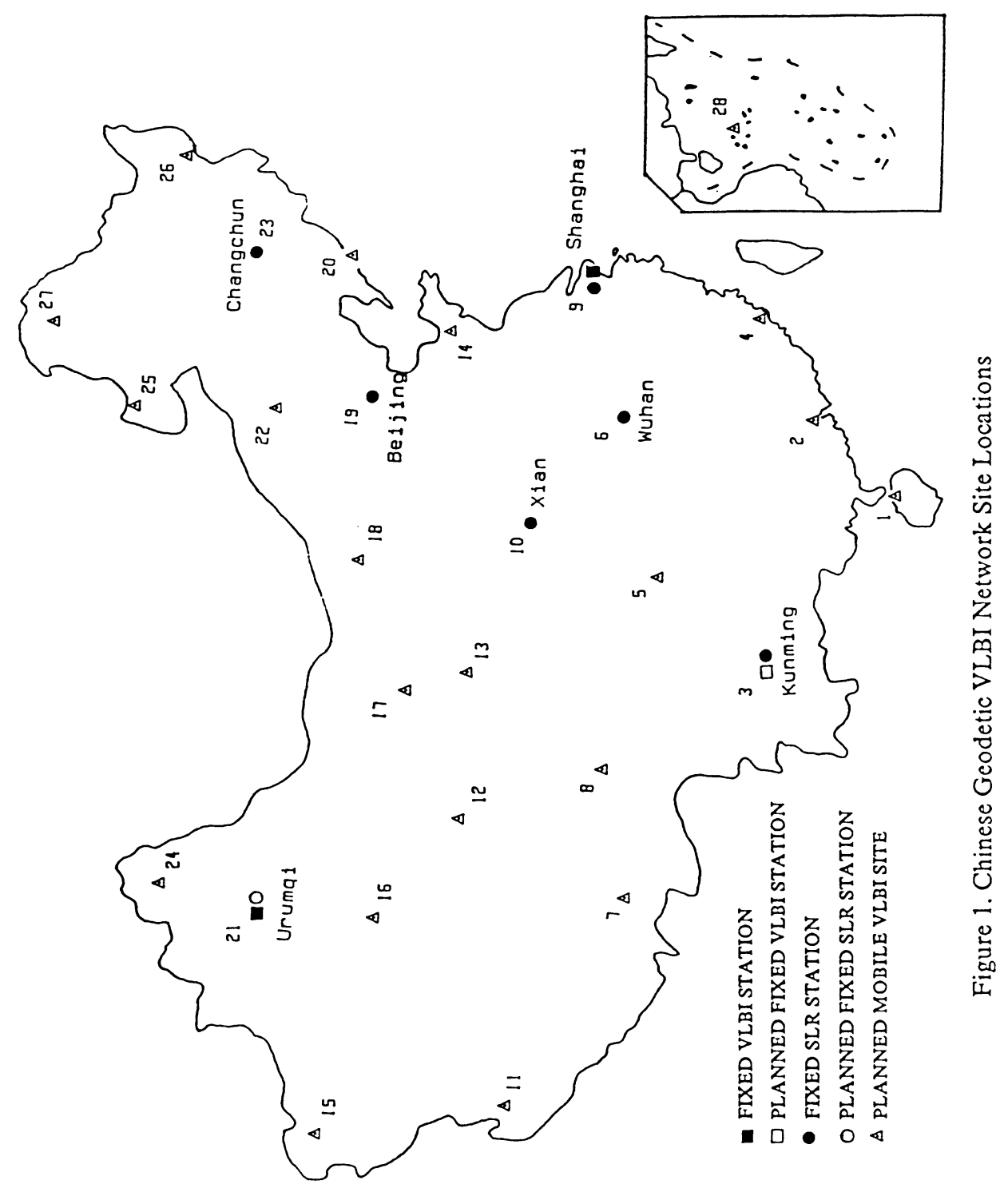

